Meta

Journal des traducteurs

Translators' Journal

\title{
Les noms d'action en tant que termes
}

\section{Ingeborga Beszterda et Jósef Sypnicki}

Volume 32, numéro 3, septembre 1987

La fertilisation terminologique dans les langues romanes

URI : https://id.erudit.org/iderudit/002070ar

DOI : https://doi.org/10.7202/002070ar

Aller au sommaire du numéro

Éditeur(s)

Les Presses de l'Université de Montréal

ISSN

0026-0452 (imprimé)

1492-1421 (numérique)

Découvrir la revue

Citer cet article

Beszterda, I. \& Sypnicki, J. (1987). Les noms d'action en tant que termes. Meta, 32(3), 255-259. https://doi.org/10.7202/002070ar d'utilisation que vous pouvez consulter en ligne.

https://apropos.erudit.org/fr/usagers/politique-dutilisation/ 


\title{
LES NOMS D'ACTION EN TANT QUE TERMES
}

\author{
INGEBORGA BESZTERDA ET JÓSEF SYPNICKI \\ Université Adam Mickiewicz, Poznan, Pologne
}

0 . Les termes scientifiques et techniques possèdent une certaine quantité de traits spécifiques (cf. M. Mazur 1961, L. Guilbert 1973, J. Sypnicki 1979 : p. 23 ss.) dont les principaux, à notre avis, sont la précision, l'univocité, la concision et la maniabilité. Aussi, puisqu'ils servent à systématiser et à hiérarchiser les notions les unes par rapport aux autres, doivent-ils se caractériser par la transparence. À ces exigences répondent, entre autres, les formations qui sont des noms d'action. Ils conviennent, on le sait bien, à traduire en premier lieu les noms de processus et de leurs résultats.

0.1 Le rôle de cette classe lexico-grammaticale est assez important - tant dans le lexique général que dans les terminologies - pour que nous soyons autorisés à attirer un peu d'attention sur elle. Nous voulons notamment faire voir la situation du français dans ce domaine et les implications lexicologiques qui en découlent. Pour ce faire, nous allons recourir à une analyse comparative des faits de la langue française et polonaise deux langues génétiquement et typologiquement assez éloignées pour que les résultats soient intéressants.

1.0 D'après l'opinion courante des linguistes et des lexicologues, les noms d'action ${ }^{1}$ constituent une catégorie quasi universelle, du moins dans les langues indoeuropéennes. Et cette opinion s'avère juste si l'on ne prend en considération que l'aspect structural et/ou si l'on pose le principe de l'homogénéité de cette classe lexicale. On peut, tout au plus, discuter sur les différences quantitatives observées entre les langues sujettes à l'analyse confrontative. En revanche, la situation devient tout à fait autre quand on étudie cette question plus à fond.

1.1. Il convient de délimiter, à l'intérieur de la classe lexicale des noms d'action, deux types qui se différencient nettement quant à leur valeur sémantique et leur fonctionnement dans le discours - à savoir les substantiva verbalia et les substantiva deverbalia (sur cette distinction fondamentale au point de vue théorique, voir J. Puzynina 1969).

1.1.1 Le premier type est constitué par les dérivés qui ne sont pas, par rapport à leurs bases, des désignations de référents nouveaux. Le suffixe y signale qu'il est question, pour des raisons syntaxiques, d'un transfert d'une catégorie lexico-grammaticale dans une autre catégorie lexico-grammaticale (partie du discours). Nous sommes donc en présence ici de la nominalisation pure et simple du verbe, sans qu'il y ait une modification sémantique quelconque par rapport à la base de dérivation.

1.1.2 Contrairement au type précédent, les nomina deverbativa formant le type suivant dénotent toujours un certain décalage sémantique plus ou moins important par rapport au sens du verbe-base (p. ex. : " résultat de l'action ", " lieu où l'action se passe ", "produit ", etc., cf. respectivement : brûlis, logement, tir).

2.0 Le premier type des noms d'action est représenté, dans la langue polonaise, par les dérivés avec le suffixe -nie (et ses variantes -enie, -scie) qui se joint pratiquement à 
n'importe quel verbe pour former une désignation nominale de l'action exprimée par ce verbe. L'élément formatif en question dispose de deux propriétés essentielles.

$2.1 \mathrm{Du}$ point de vue sémantique, il y a une équivalence parfaite avec le sens du verbe-base, en conséquence de quoi, on ne constate pratiquement aucun écart par rapport à la base de dérivation ni une tendance à la lexicalisation de ces formations; rares sont les cas où, à côté de la valeur " action verbale indiquée par le verbe - base " fonctionnent également les sens dérivés (p. ex. : rozdarcie - " action de déchirer " et " déchirure ") - toutefois, même ici, le sens catégoriel dominera toujours et il sera considéré par le sujet parlant comme sens primaire.

2.2 Les formations en question jouissent, au point de vue morphologique, d'une telle productivité en polonais qu'on pourrait les traiter comme faits de flexion et non pas de dérivation (l'adjonction du suffixe -(e)nie / -cie n'est impossible que pour un groupe restreint de verbes modaux et de verbes impersonnels du polonais). En français, à cela n'est comparable que la nominalisation des infinitifs au moyen de l'article défini - et, pour être plus précis, les nominalisations des infinitifs qui se faisaient en ancien français, car en français moderne ce procédé semble n'avoir plus un caractère spontané. De plus, l'infinitif nominalisé ne peut fonctionner dans les fonctions obliques, ni avoir des compléments (bien entendu, les formes lexicalisées du type dîner, devoir, etc., mises à part). Â la lumière des faits susmentionnés, nous pouvons poser l'absence des substantifs verbaux dans la langue française d'aujourd'hui.

3. Quant au deuxième type des noms d'action (substantifs déverbatifs), les deux langues analysées semblent, à première vue, s'équivaloir. Elles disposent, l'une et l'autre, de longues séries d'éléments formations qui servent à construire des dérivés où l'on retrouve la valeur sémantique plus ou moins nette de l'«action indiquée par le verbebase ", mais dominée par d'autres valeurs dérivées de celle-ci. Sous l'angle sémantique, le polonais s'écarte ici du français, surtout par la possibilité de traduire l'attitude affective du sujet parlant envers l'action énoncée. À titre d'exemple harówa (" travail dur ") a un caractère augmentatif, les formations avec le suffixe -ina ont le schéma sémantique "l'action chaotique indiquée par le verbe-base" (p. ex. : bieganina), les dérivés en -iny sont pourvus d'un caractère officiel pour ainsi dire (type odwiedziny), par contre, les dérivés avec le suffixe - $k a$ sont sentis comme populaires ou argotiques (wpadka, odsiadka, etc.) (cf. R. Grzegorczykowa $1984: 33$ ). Il serait difficile de trouver des procédés analogues en français, sauf, peut-être, les formations avec -erie et -ade.

4.0 On note également des différences notables quand on analyse le côté formel du problème. Il existe en premier lieu des différences quantitatives résultant du fait qu'en français les verbes constituent une classe numériquement assez modeste - surtout par rapport au polonais, ainsi que de la productivité restreinte des moyens dérivationnels suffixaux du français.

4.1 D'autre part, bien que le français s'avère une langue plutôt pauvre en verbes comme nous l'avons signalé plus haut, nombre d'entre eux ne possèdent pas de noms d'action (A. Sauvageot $1984: 143$, cite entre autres : garer, traquer, attraper, etc.) et d'autres disposent de noms d'action empruntés à la langue latine, p. ex. : boire - bibition, tirer - traction, voir - vision (id. ib.) où la valeur primaire du nom d'action s'efface la plupart du temps devant des emplois plus ou moins spécialisés : manducation estil vraiment le nom d'action de manger? - où plutôt un terme de physiologie ?

5.0 Quand on aborde l'aspect chronologique du problème en français, on s'aperçoit vite que la presque totalité des noms d'action sont des formations relativement an- 
ciennes. Ce fait s'explique sans doute d'une double manière; ils constituent une classe de dérivés qui se sont montrés vite nécessaires à la communication - ils apparaissent donc très tôt dans le lexique (à rappeler que les besoins communicatifs font naître également aujourd'hui des formations du type transfèrement ( $=$ transfert), transportage, transportement ( $=$ transport) (A. Sauvageot $1964: 141$ ), mais ce phénomène s'explique aussi par le fait que la langue moderne évite plutôt de former de nouveaux noms d'action : la preuve en sont les données de Jean et Claude Dubois (1971). Ces auteurs - il y a lieu de le rappeler - constatent (1971:114-115) pour -age 75 entrées et 121 sorties et pour -ment - 41 entrées contre 152 sorties. Ils en concluent que la langue tend à réduire le nombre des doublets synonymiques. Ensuite, les auteurs ont trouvé 105 cas où le dérivé en -age ou en -ment subsiste seul et 41 cas où l'un deux cède la place à l'autre. On n'observe pas de préférences visibles dans le choix du suffixe, car l'usage élimine d'une part affichement, polissement et de l'autre achevage, apparentage, etc. (se maintiennent respectivement affichage, polissage, achèvement, apparentement). Qui plus est, les auteurs cités ont noté également la tendance de la langue à remplacer les dérivés traditionnels en -age ou -ment par les formations en -tion et ses variantes (bien qu'il y ait, pour ce dernier suffixe, un équilibre relatif entre les entrées et les sorties - même ici les éliminations dominent / notamment 109 entrées contre 122 disparitions). Les auteurs expliquent cet état de choses par l'élimination des termes techniques désuets. Cela n'explique pourtant, à notre avis, qu'une partie du problème ; la raison de cette tendance a sans doute un caractère intralinguistique (nous pensons ici à la désintégration des familles de mots en français et, par conséquent, à la productivité restreinte de la dérivation). De même, la spécialisation sémantique actuelle entre -age et -ment (actions de caractère abstrait vs actions concrètes) ne modifie que très peu cet état de choses, puisque la lexicalisation rapide des dérivés et leur spécialisation sémantique détruisent cette régularité (p. ex. : barrement [pour les chèques], ailleurs barrage).

5.1 A. Sauvageot semblait voir la « faiblesse " des déverbatifs - noms d'action du français dans la "... totale indétermination de nos substantifs verbaux (qui) fait contraste avec la rigoureuse distinction observée dans d'autres langues" (A. Sauvageot $1964: 145$ ). Toutefois, il convient de signaler que la langue polonaise également ne fait pas de distinction ici entre le passif, l'actif et le réfléchi - du moins d'une manière systématique et régulière (car on trouve des cas du type pochylanie / pochylanie sie) et que les syntagmes du type "druk ksiazki» / "impression du livre», "wybór prezidenta» / "élection du président " entraînent la même ambiguïté dans les deux langues et pour la résoudre, elles recourent à des procédures analogues. De même, le sujet parlant polonais ne se sent pas gêné dans l'emploi des noms d'action bien qu'ils soient aussi comme ceux du français - non localisés dans le temps, puisque, dans le discours, les noms d'action assurent une telle concision de l'énoncé qu'elle récompense pleinement, le contexte aidant, les inconvénients évoqués plus haut.

6.0 Nous avons soumis à l'analyse l'article A dans le Dictionnaire du français contemporain. Pour un total de 121 formations que l'on peut qualifier de noms d'action, la source lexicographique en question admet la possibilité d'emploi en tant qu'action verbale nominalisée, pour presque la totalité de ces formations. Mais cela n'est qu'une possibilité théorique plutôt, puisque l'observation des faits du discours nous convainc que de tels emplois sont rarement réalisés sur le sujet parlant. Par ailleurs, il est clair que la valeur de " nomen verbale » n'apparaît souvent que dans les définitions lexicales - héritées de la tradition lexicographique et répétées plus ou moins automatiquement; l'exemplification fournie par les dictionnaires est assez significative à ce point de vue. En conséquence de quoi, une quantité considérable de ces noms d'action du français ne 
base. peuvent être reconnus pour les nominalisations du verbe-base. Autrement dit, la paraphrase lexicale "le fait de brûler " ne peut pas rendre compte du dérivé du type "brûlis ".

6.1 Il découle de ce qui précède que le lexique du français, dans le domaine analysé, démontre la déficience des nomina actionis résultant tant de la modicité numérique de la classe des verbes que de la tendance à la lexicalisation et à la spécialisation des dérivés. Cet état de choses a des conséquences au niveau syntaxique vu la rareté des substantiva verbalia, mais aussi au niveau lexical où l'usager de la langue se voit obligé de recourir - pour satisfaire à ses besoins communicatifs dans le domaine des terminologies surtout - à des emprunts ou à des éléments formatifs d'origine étrangère.

6.2 Les formations avec le suffixe -tion constituent, il nous semble, l'exemple le mieux choisi pour illustrer le processus en question. Le français y recourt systématiquement depuis ses origines, pour combler les lacunes dans le lexique dues à l'insuffisance des verbes. Bien entendu, les dérivés décrits appartenaient toujours au domaine des terminologies plus ou moins spécialisées.

Nous avons examiné l'article A dans le Petit Larousse. Nous y avons trouvé 147 unités lexicales où l'élément -tion est aisément détachable tant du point de vue morphologique que sémantique. Sous l'angle chronologique, elles se répartissent comme suit :

\begin{tabular}{|c|c|}
\hline $\mathrm{XI}^{\mathrm{e}} \mathrm{S} .-1$ & XVI' s. -19 \\
\hline XII'e s. -14 & $\mathrm{XVII}^{\mathrm{e}} \mathrm{s} .-2$ \\
\hline $\mathrm{XIII}^{\mathrm{e}}$ s. -21 & XVIII' s. - \\
\hline $\begin{array}{l}\text { XIVe s. }-28 \\
\text { XVe s. }-12\end{array}$ & $\begin{array}{l}\mathrm{XIX}^{\mathrm{e}} \mathrm{s} . \\
\mathrm{XX}^{\mathrm{e}} \mathrm{s} . \\
\end{array}$ \\
\hline
\end{tabular}

À remarquer que, pour le XXes., 16 formations ne sont que des préfixés au moyen de l'élément auto- joint à des bases qui fonctionnent déjà dans le lexique avant le $\mathrm{XX}^{\mathrm{e}} \mathrm{s}$. Le chiffre réel des formations nouvelles avec -tion pour le $\mathrm{XX}^{\mathrm{e}} \mathrm{s}$. n'atteint donc que 26 dérivés.

6.3 Les données présentées plus haut se répartissent au point de vue chronologique d'une manière assez analogue à la répartition chronologique des latinismes en français. Nous voyons que la langue contemporaine - vu le développement des sciences et des techniques - se sert relativement peu de ce procédé dérivationnel. Pourtant le suffixe en question - comme il fonctionne dans toutes les langues romanes et n'est pas étranger à l'anglais, peut servir à la "fertilisation 》 du lexique et se prête bien à une bonne internationalisation de la communication.

6.4 Par ailleurs, nous voulons attirer également l'attention sur un type particulier de la francisation des mots d'emprunt, et que peut assumer les deux fonctions précitées. Nous pensons notamment à la subtitution que la langue courante fait du suffixe-ing par le suffixe -age, cf. : antidoping - antidopage, aquaplaning - aquaplanage, etc.

7. En concluant nos remarques sur les nomina actionis, nous pouvons affirmer que la langue française dispose des moyens dérivationnels traditionnels à caractère « terminogène ", aptes à assurer, sans déformer la langue, une bonne internationalisation de la communication. C'est le cas, sans doute, de nombreux suffixes dits " savants " fonctionnant également dans d'autres langues romanes et retrouvables en anglais et en allemand - en premier lieu du suffixe -tion qui fournit des dérivés à structure sémantique et morphologique transparente et, de plus, assurant, dans certains de ses emplois, une concision remarquable au niveau syntaxique. Sans chercher donc des procédures dérivationnelles nouvelles, peu connues pour le sujet parlant moyen, on pourrait mettre à profit les moyens vérifiés par la tradition. 


\section{BIBLIOGRAPHIE}

DOROSZEWSKI, W. (1970) : Elementy leksykologii i semiotyki, Warszawa, PWN.

DAUZAT, A., J. DUBOIS, H. MITTERAND (1971) : Nouveau dictionnaire étymologique et historique, Paris, Larousse.

Dictionnaire du français contemporain (1966) : Paris, Larousse.

DUBOIS, Jean (1962) : Étude sur la dérivation suffixale en français moderne et contemporain, Paris, Larousse. DUBOIS, Jean et Claude DUBOIS (1971) : Introduction à la lexicographie : le dictionnaire, Paris, Larousse. GRZEGORCZYKOWA, R. (1984) : Zarys slowotwórstwa polskiego, Sllc/owotwórstwo opisowe, Warszawa, PWN.

GUILBERT, Louis (1965) : la Formation du vocabulaire de l'aviation, Paris, Larousse.

GUILBERT, Louis (1975) : la Créativité lexicale, Paris, Larousse.

HONOWSKA, M. (1967) : Zarys klasyfikacji derywatów, Wroclaw.

LERAT, Pierre (1984) : "Grammaire des noms d'agents en -ant en français contemporain ", in Cahiers de lexicologie, pp. 23-39.

MAZUR, M. (1961) : Terminologia techniczna, Warszawa.

Petit Larousse en couleurs (1983).

PUZYNINA, J. (1969) : Nazwy czynności w jezyku polskim, Warszawa.

SAUVAGEOT, A. (1964) : Portrait du vocabulaire français, Paris.

SYPNICKI, J. (1979) : la Composition nominale en français et en polonais, Poznań, UAM. 


\title{
LES SYNTAGMES NOMINAUX COMPLEXES EN ANGLAIS ET EN FRANÇAIS : ÉLÉMENTS DE RÉFLEXION
}

\author{
MARIE-Josée JASTRAB DE SAINT-RobERT \\ New York University, USA
}

Cet article se propose d'aborder le problème de l'incidence de l'anglais ${ }^{1}$ sur le français par le biais de l'étude de la formation des syntagmes nominaux complexes dans ces deux langues. Sont considérés comme des syntagmes nominaux complexes les mots composés lexicalisés ou en voie de lexicalisation et les séquences nominales produites par les règles syntactiques ou sémantiques mais qui ne seraient pas présentes telles quelles dans le lexique. Parmi les mots composés nous retiendrons en particulier les mots composés complexes, tels que :

(1) a. input/output/device organe entrée/sortie

b. split screen écran multifenêtre

c. cash flow marge brute d'autofinancement

d. voice changer modulateur de voix

Les séquences nominales ordinaires qui nous intéresseront ici seront essentiellement des séquences composées de noms qualifiant d'autres noms :

(2) a. customer information

b. letter quality printer

bureau

bureau d'information-client imprimante qualité courrier

Du point de vue terminologique, ces deux types de syntagmes qui correspondent à des unités de syntaxe et de signification constituent des termes à part entière. Plusieurs questions seront au centre de notre réflexion : y-a-t-il influence de l'anglais sur le français dans le domaine de la formation de ces syntagmes? N'y-a-t-il pas en français des règles susceptibles de produire de tels énoncés? Autrement dit, peut-on véritablement parler de fertilisation terminologique du français par l'anglais dans le domaine des constructions nominales?

Pour répondre à ces questions il nous faut tout d'abord définir ce que nous entendons par fertilisation terminologique. Cette notion peut en effet recouvrir différentes réalités. Ainsi, la fertilisation terminologique peut être considérée comme synonyme de fertilisation notionnelle. Comme la démarche terminologique a pour point de départ la notion et non le terme, il y a fertilisation terminologique dès lors qu'il y a importation d'une notion nouvelle exprimée d'abord en anglais puis reprise en français. Le syntagme research and development ( $R$ and $D$ ), d'abord apparu en anglais dans la terminologie militaire, de même que la notion qu'il exprime, a donné lieu à nombre d'équivalents français : élaboration et mise au point, étude et mise au point, recherche et mise au point avant de devenir recherche-développement (la R-D). Ce type de fertilisation terminologique est d'autant plus difficile à repérer que les spécialistes du domaine ne mentionnent pas toujours la source ou la langue source des notions qu'ils manipulent. Le terminologue doit donc consulter ou être lui-même un spécialiste du domaine pour pouvoir déter- 